\title{
Threat detection of liquid explosives and precursors from their x-ray scattering pattern
} using energy dispersive detector technology

\author{
Kehres, Jan; Olsen, Ulrik Lund; Lyksborg, Mark
}

Published in:

Proc. SPIE 10403, Infrared Remote Sensing and Instrumentation XXV

Link to article, DOI:

$10.1117 / 12.2274519$

Publication date:

2017

Document Version

Publisher's PDF, also known as Version of record

Link back to DTU Orbit

Citation (APA):

Kehres, J., Olsen, U. L., \& Lyksborg, M. (2017). Threat detection of liquid explosives and precursors from their xray scattering pattern using energy dispersive detector technology. In Proc. SPIE 10403, Infrared Remote Sensing and Instrumentation XXV (Vol. 10391). [1039302] SPIE - International Society for Optical Engineering. Proceedings of SPIE - The International Society for Optical Engineering https://doi.org/10.1117/12.2274519

\section{General rights}

Copyright and moral rights for the publications made accessible in the public portal are retained by the authors and/or other copyright owners and it is a condition of accessing publications that users recognise and abide by the legal requirements associated with these rights.

- Users may download and print one copy of any publication from the public portal for the purpose of private study or research.

- You may not further distribute the material or use it for any profit-making activity or commercial gain

- You may freely distribute the URL identifying the publication in the public portal 


\section{Threat detection of liquid explosives and precursors from their x-ray scattering pattern using energy dispersive detector technology}

Jan Kehres, Mark Lyksborg, Ulrik L. Olsen

Jan Kehres, Mark Lyksborg, Ulrik L. Olsen, "Threat detection of liquid explosives and precursors from their $\mathrm{x}$-ray scattering pattern using energy dispersive detector technology," Proc. SPIE 10393, Radiation Detectors in Medicine, Industry, and National Security XVIII, 1039302 (7 September 2017); doi: $10.1117 / 12.2274519$

SPIE Event: SPIE Optical Engineering + Applications, 2017, San Diego, California, United States 


\title{
Threat detection of liquid explosives and precursors from their $x$-ray scattering pattern using energy dispersive detector technology
}

\author{
Jan Kehres ${ }^{* a}$, Mark Lyksborg ${ }^{\mathrm{b}}$, Ulrik L. Olsen ${ }^{\mathrm{a}}$ \\ ${ }^{a}$ Technical University of Denmark, Department of Physics, B 307, Fysikvej, 2800 Kgs. Lyngby, \\ Denmark, ${ }^{b}$ Technical University of Denmark, Department of Applied Mathematics and Computer \\ Science, B 324, Richard Petersens Plads, 2800 Kgs. Lyngby, Denmark
}

\begin{abstract}
Energy dispersive X-ray diffraction (EDXRD) can be applied for identification of liquid threats in luggage scanning in security applications. To define the instrumental design, the framework for data reduction and analysis and test the performance of the threat detection in various scenarios, a flexible laboratory EDXRD test setup was build. A data set of overall 570 EDXRD spectra has been acquired for training and testing of threat identification algorithms. The EDXRD data was acquired with limited count statistics and at multiple detector angles and merged after correction and normalization. Initial testing of the threat detection algorithms with this data set indicate the feasibility of detection levels of $>95 \%$ true positive with $<6 \%$ false positive alarms.
\end{abstract}

Keywords: Threat detection, Liquid threats, LAGs, EDXRD

\section{INTRODUCTION}

After the transatlantic Airplane plot in 2006 [1] the maximum volume of liquids, aerosols and gels (LAG's) in carryon luggage was temporarily limited to 1 Liter, packed in individual bottles of max. $100 \mathrm{ml}$, until scanner technology emerges capable of identifying liquid threats. With conventional scanners, performing dual-energy radiography, the atomic number $Z$ and the density $\rho$ can be retrieved and used for identification of threats and illicit substances [2], however, with a considerably high number of misclassifications due overlapping objects and vague approximation of the object thickness. A more accurate approach for threat material identification in security applications is inspecting the material using diffracted x-rays [3]-[11]. Firstly the diffraction pattern, arising from interference of the coherently scattered x-rays in the volume element under inspection, is unique and can be interpreted as a fingerprint more accurate than attenuation contrast. The higher accuracy of the method leads to fewer manual inspections. Secondly due to the large penetration power of $\mathrm{x}$ rays, material identification of suspicious objects by diffraction can be performed without requiring removal of the object from the luggage. With fewer tasks for both security officer and passenger the efficiency of the security screening is consequently increased using EDXRD.

The scattering probability for the x-rays is low and strict collimation is required resulting in low count statistics, however this can be improved by the use of 2D detectors [3]. Scanning of the whole luggage in time frames realistic for security applications with a simple pencil beam geometry and a point detector is not feasible. The scanning time has been reduced by either pre-identifying suspicious objects with conventional transmission x-ray scanning [4] or by implementation of elaborated multi-source- detector-geometries to perform diffraction imaging of the whole luggage [5]-[7].

X-ray diffraction is commonly used to study crystalline materials and can be conceptually explained by Bragg's law $n \lambda=2 d \sin (\theta / 2)$, where $\lambda$ is the wavelength. The $x$-rays are scattered by atoms ordered periodically in crystal planes which are separated by a spacing $d$. Constructive interference occurs if the path difference between the scattered waves from the successive crystal planes is an multiple integer of $\lambda$ and signal and scattering angle $\theta$ in respect to the crystal plane. Diffraction patterns of crystalline materials show a number of sharp reflections, with the position and height related to the microscopic structure and elemental composition. Although amorphous materials and liquids have no periodic structure as crystals, they exhibit a near range order which is dependent on interaction between the molecules and density. The structure of the liquid can be described by pair-wise radial distribution functions, which for each atom describes the probability

\footnotetext{
*jake@fysik.dtu.dk; phone +45 5337 1215; fysik.dtu.dk
}

Radiation Detectors in Medicine, Industry, and National Security XVIII, edited by Gary P. Grim, Lars R. Furenlid, H. Bradford Barber, Proc. of SPIE Vol. 10393, 1039302 · @ 2017 SPIE CCC code: $0277-786 X / 17 / \$ 18 \cdot$ doi: $10.1117 / 12.2274519$ 
density of another atom being in a certain distance. With increasing distance the probability function quickly tends to unity and as a consequence diffraction patterns of liquids show a rather broad reflection signal compared to crystalline materials. The application of X-ray diffraction for detection of liquid threats in security application is currently investigated [7]-[9].

For analytical applications X-ray diffraction is usually performed using monochromatic X-rays and recording the diffracted signal as a function of scattering angle, this is also termed angular dispersive x-ray diffraction (ADXRD). Currently room temperature CdZn and CdTeZn detectors are emerging. These detectors are exhibiting superior detection efficiency in the energy window of $\mathrm{x}$-rays used for luggage screening compared to silicon. This development makes it feasible to collect the whole diffraction spectrum at one or more angles. This corresponds to the method named EDXRD. Although the limited energy resolution of the room temperature detectors yields broader peaks compared to ADXRD, acquisition times are significantly shorter due to the use of a larger fraction of the emitted spectrum. The data in this work is presented as a function of the scattering vector with the magnitude:

$$
Q=\frac{4 \pi E}{h c} \sin (\theta)
$$

where $h$ is the Plank constant, $c$ the vacuum velocity of light and $E$ the energy of the x-ray photon. Here should be mentioned that the scattering vector is also often expressed as $x=\sin (\theta) E / h c$.

In this manuscript the experimental setup used for acquisition of EDXRD pattern with limited count statistics and at multiple detector angles is presented. Furthermore the required post processing of the data and results from a first classification experiment for the liquid samples are shown.

\section{EXPERIMENTAL SETUP}

The experiments were performed in a non-commercial and in-house developed energy dispersive $\mathrm{x}$-ray diffractometer, located in the Department of Physics at the Technical University of Denmark, a 3D sketch of the setup is shown in Figure 1.

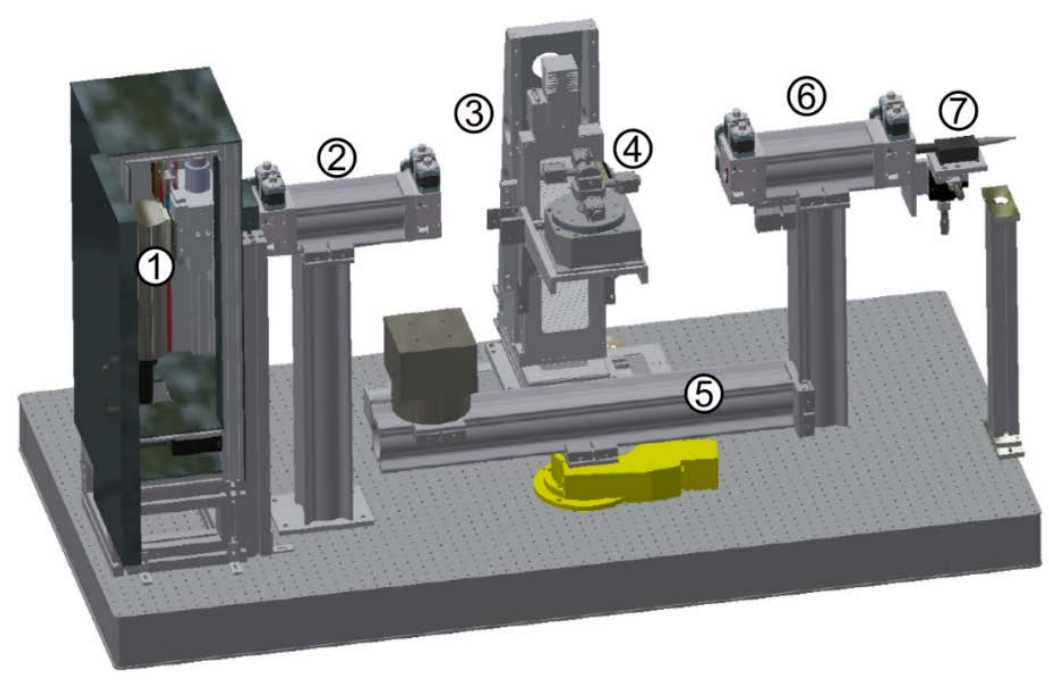

Figure 1. 3D drawing of the EDXRD test setup X-ray tube with (1) installed in an extra shielding, the primary collimator

(2) consisting of two slits with $5 \mathrm{~mm}$ W-blades and a flight tube, the XYZ sample manipulation (3) with mounted goniometer (4), the detector arm (5), the secondary collimator (6) consisting of similar components than the primary collimator and the energy dispersive 0D detector (7).

The x-rays are generated by an industrial metal-ceramic tube of the type MXR-160HP/11 and a high voltage generator of type XRP-160/2250/2 (Comet AG, Flamat, Switzerland). Both primary and scattered beam are collimated by an assembly of two pairs of X-ray slits of the type IB-C30-Air (JJ X-Ray A/S, Hørsholm, Denmark) with $5 \mathrm{~mm}$ thick tungsten blades. 
The transmitted and scattered beam spectra are recorded with a solid state detector of the type XR-100T-CdTe and PX-5 pulse processor (Amptek Inc., Bedford, United States). The detector arm is mounted on a rotation stage for adjusting the scattering angle. Samples with a weight up to $8 \mathrm{~kg}$ can be translated $100 \mathrm{~mm}$ in horizontal, $250 \mathrm{~mm}$ in vertical and in beam direction. All components of the experimental setup are controlled and the data logging is performed by the scientific instrument control software SPEC [12].

For precise positioning of small samples a motorized goniometer head of the type 1003-MS (HUBER Diffraktionstechnik GmbH \& Co. KG, Rimsting, Germany) can be used optionally, see Figure 2 left. This configuration describes the instrument used for EDXRD. The setup can easily be rearranged to perform energy dispersive radiography and tomography, when removing the second slit of the primary beam collimator and placing 2 Multix ME100 line detectors [13] in the detector holder box shown in Figure 2 right.
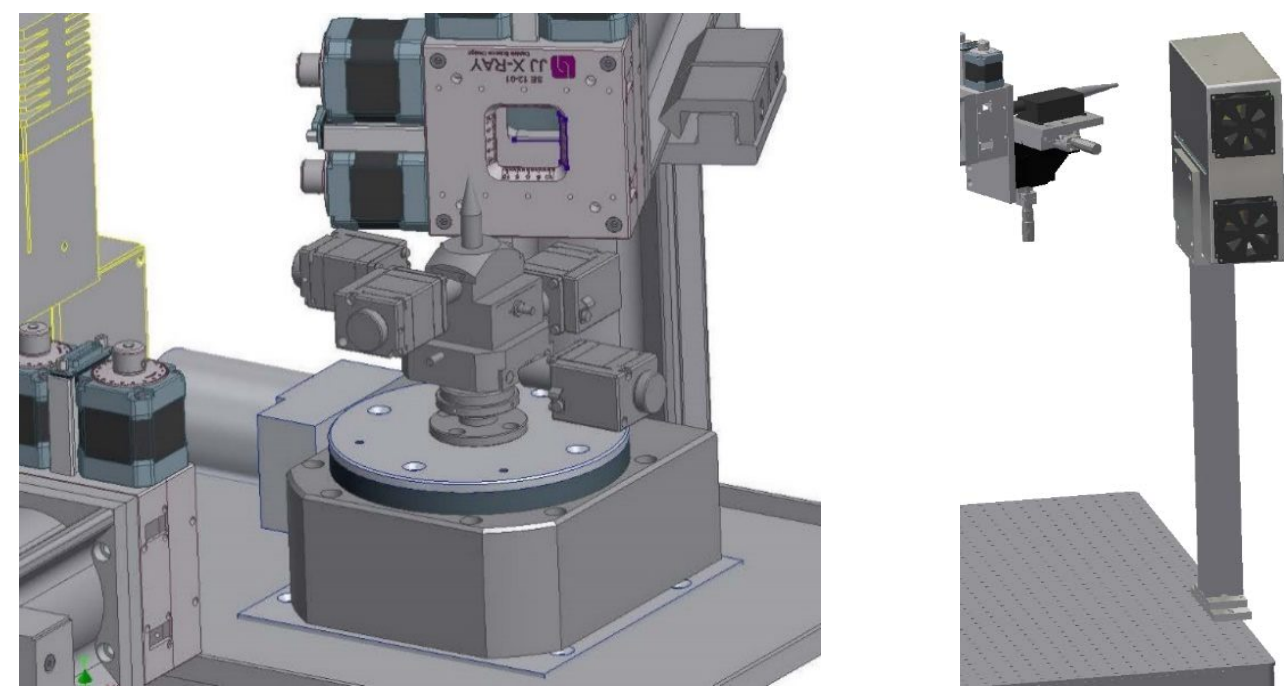

Figure 2. 3D drawing of the motorized goniometer head (left) and the holder for 2 Multix ME100 line detectors (right)

\section{DATA ACQUISITION}

For the development of classification algorithms we acquired a library of 570 EDXRD patterns consisting of 11 liquid precursors for synthesis of explosives and 46 commonly found liquids. Each EDXRD pattern consists of 10 spectra recorded at scattering angles $2 \theta$ between $2^{\circ}$ and $6.5^{\circ}$ in steps of $0.5^{\circ}$. The test data was acquired with statistics of 1000 counts per $2 \theta$ and 72000 counts for the transmitted beam at $2 \theta=0^{\circ}$. Each measurement was repeated for 10 times to get statistical significant results for the classification. Furthermore we acquired a reference data set of the 11 threats with $>500000$ counts per $2 \theta$. All samples were investigated in the original packing.

The experiments were performed with an incident beam size at the second slit of the primary collimator of $1 \mathrm{x} 5 \mathrm{~mm}$ (width $\mathrm{x}$ height). The width of the slits of the secondary collimator was readjusted at every setting of $2 \theta$ to define a scattering volume which only intersected with the inside of the container while maintaining the highest possible count rate. The height of the slits were kept at $5 \mathrm{~mm}$, comparable to the crystal size of the detector. Transmitted beam spectra at $2 \theta=0^{\circ}$ were acquired with a pinhole formed by the second slit of the secondary collimator, keeping the count rate $<12000 \mathrm{cs}^{-1}$ and thereby in the dynamic range of the detector.

\section{DATA PROCESSING}

A framework for the data reduction was implemented using Matlab ${ }^{\circledR}$ for batch processing of the acquired data sets. The steps of the data processing is explained in the following for a reference data set of hydrogen peroxide with a concentration of $w(\mathrm{H} 2 \mathrm{O} 2)=30 \%$. 
(a)

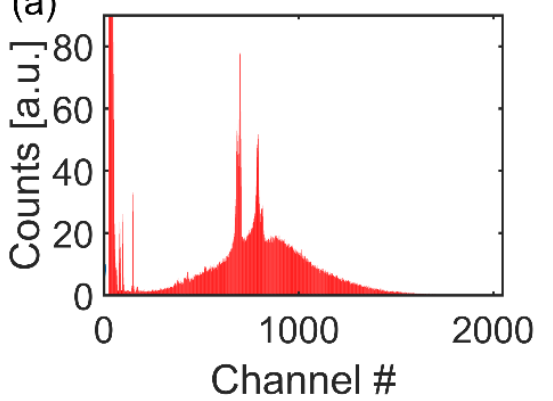

(d)

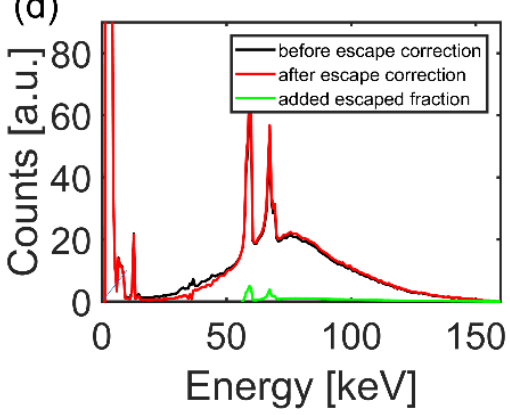

(b)
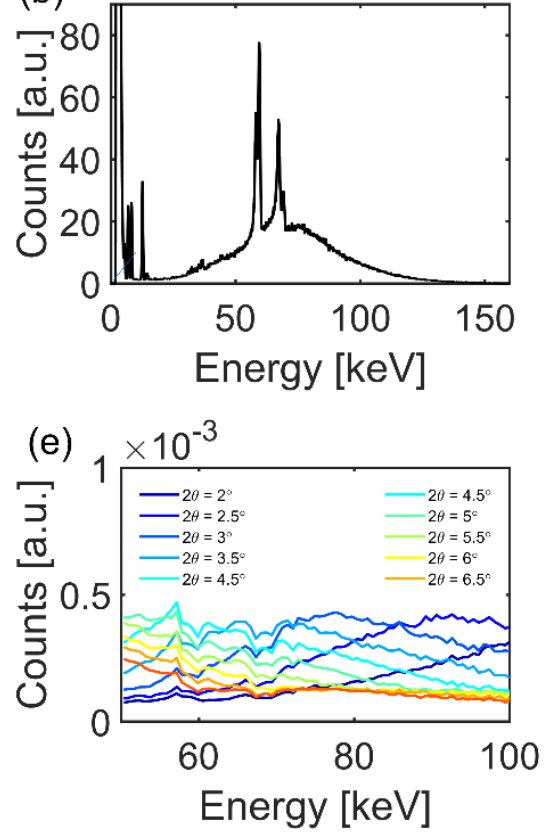

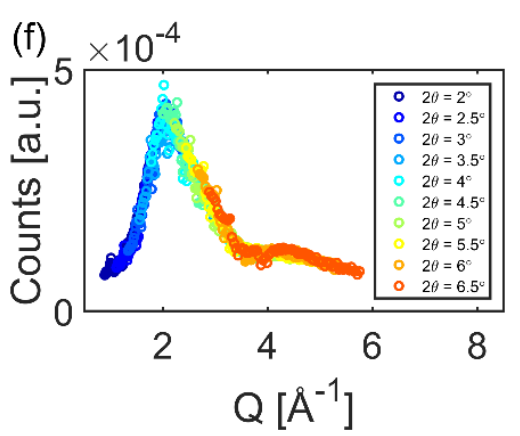

(c)

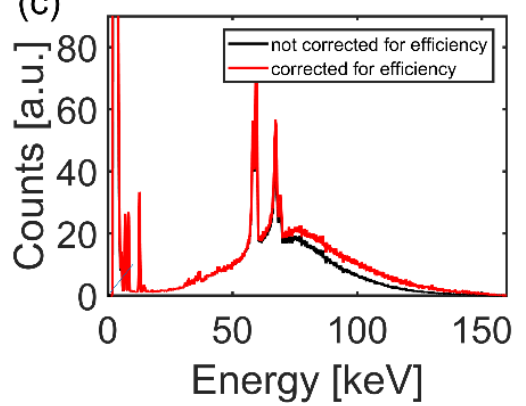

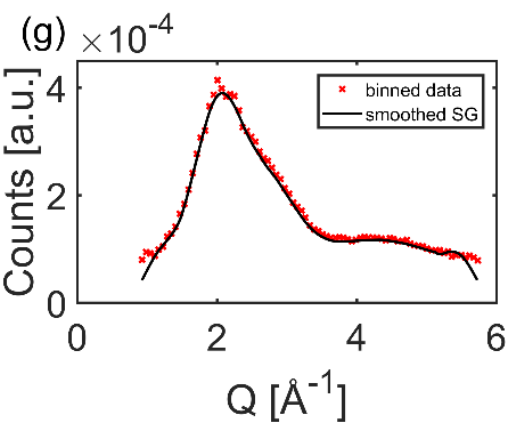

Figure 3. Data preprocessing structure of the EDXRD data demonstrated on a measurement of a reference sample of hydrogen peroxide $w\left(\mathrm{H}_{2} \mathrm{O}_{2}\right)=30 \%$ with histogram of the raw data at $2 \theta=3.5^{\circ}$, (b) plot as a function of energy, (c) correction for detector efficiency, (d) escape correction, (e) scaling of data from multiply angles and normalization to transmitted beam, (f) transforming to modulus as function of $Q$ and overlapping data acquired at multiple angles and (g) binning and smoothing of data.

Each channel of the histogram (Figure $3 \mathrm{a}$.) is related to an energy (Figure $3 \mathrm{~b}$.) using the calibration coefficients determined from linear regression of the centroids of the channels for the $\mathrm{W}-\mathrm{K} \alpha$ and the $\mathrm{W}-\mathrm{K} \beta$ lines. Correction for the detector efficiency is performed with the data provided from the manufacturer (Figure $3 \mathrm{c}$.). The data is corrected for escape events by a stripping algorithm (Figure 3 d.) reported in [14]. Afterward the data collected at all $2 \theta>0^{\circ}$ is normalized by the transmitted beam spectrum and scaled by the scattering volume calculated from the geometry (Figure 3 e.). The data is transformed to a function of $Q$ using eq. 1 (Figure $3 \mathrm{f}$.). Then the data is binned and smoothed using a Savitzky-Golay filter with a cubic function (Figure 3. g.) [15].

\section{RESULTS}

To inspect the validity of the data acquired in our laboratory EDXRD we compared it to data of similar materials acquired with ADXRD at the synchrotron beamline ID9 at ESRF. In Figure 4 a plot of diffraction patterns acquired with both instruments is shown. Comparable diffraction patterns are acquired for water and ethanol. 

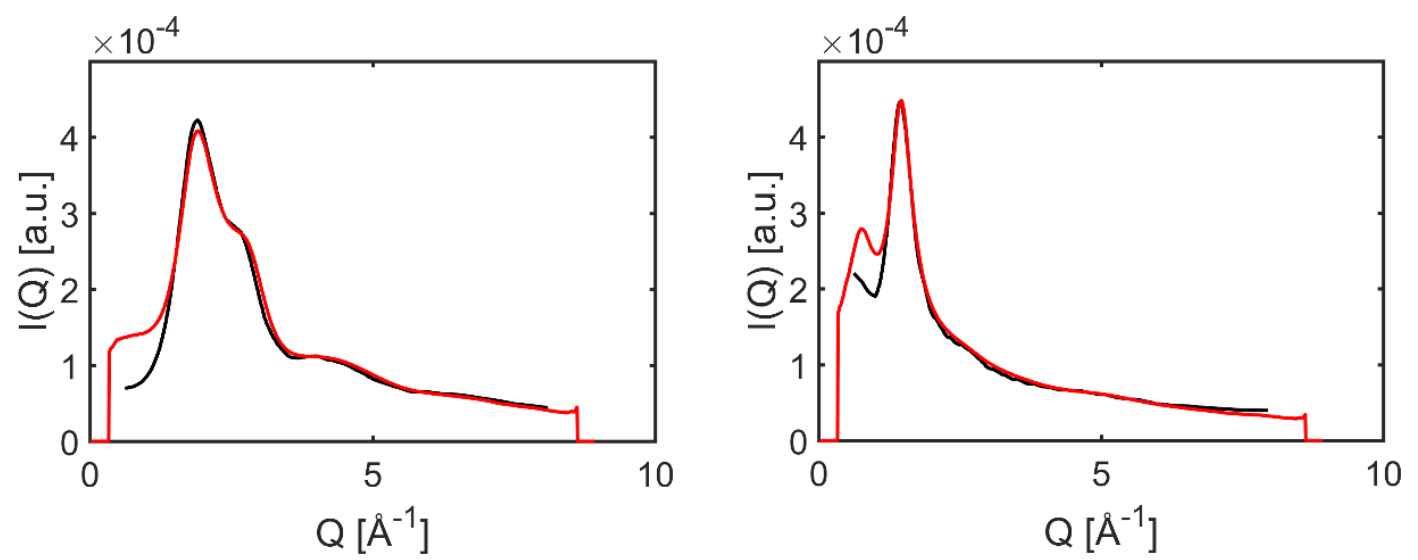

Figure 4. EDXRD pattern of water (left) and ethanol (right) acquired in the in-house setup (black lines) plotted together with ADXRD patterns acquired at ID09 at ESRF,

The diffraction patterns acquired for water with ADXRD at a Synchrotron source and from our laboratory setup show good agreement at scattering vectors $>1 \AA^{-1}$. The higher intensity at low $Q$ for the ADXRD acquired at the Synchrotron source compared to the EDXRD can be explained in the following. The EDXRD data was acquired from a sample in a PE-bottle with a diameter $d=68 \mathrm{~mm}$ surrounded by air and with the slits adjusted to define a gauge volume solely intersecting with the inside of the sample container. The synchrotron data was recorded from a sample with a sheet thickness of $0.3 \mathrm{~mm}$ in a liquid jet setup, the liquid sheet itself is protected in a sample cell closed with 2 Kapton ${ }^{\circledR}$ x-ray windows and a protective atmosphere of argon. The synchrotron setup thus has a lower interaction volume in the liquid and a lower signal to airscattering ratio compared to the EDXRD. Furthermore the synchrotron experiment has additional scattering from the Kapton ${ }^{\circledR}$ windows and the larger atmospheric contribution due to the larger form factor of argon compared to air.

In realistic luggage screening applications the duration of the inspection is a crucial factor. For security application the acquisition of the data for threat identification has to be performed in a time frame of a few seconds, which yield very limited count statistics. Rather than using acquisition time as a parameter we have chosen to use the number of counts. For classification the number of counts defines the quality of the EDXRD spectra. Conversely the correspondence between number of counts and acquisition time is not straight forward. Firstly the scattered intensity at different detector angles varies depending on the near range order and composition of the liquids and secondly the width of the secondary collimator the liquids were adjusted to maximize the interaction volume for different sizes of sample containers and at the scattering angles the data was acquired.

To compare the effect of the number of acquired photon counts, EDXRD spectra with $1000-10000$ counts per $2 \theta$ is shown in Figure 5 together with the reference spectrum acquired with $>500000$ counts per $2 \theta$. Visually good agreement of the scattering patterns acquired with 1000 - 10000 counts with the reference sample can be seen, at $Q<4 \AA^{-1}$ the data acquired with 1000 counts per $2 \theta$ already seems to represent all features of the water reference data. Experiments for the classification test were therefore performed with 1000 counts per $2 \theta$. The motivation of this work is the use of EDXRD in an airport luggage screening application. This leads to an aim for an acquisition time of $\sim 6$ s per threat identification. The transmitted beam spectrum required for normalization of the scattered beam spectra can therefore be acquired in the same time frame with 72000 counts keeping the count rate in the dynamic range of the XR-100T-CdTe detector. 


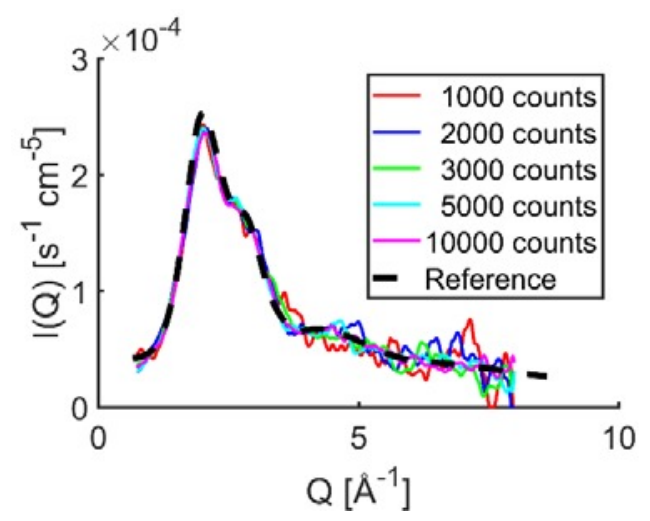

Figure 5. Overlapped EDXRD of the water acquired with 1000 - 10000 counts per $2 \theta$, the data has been smoothed by a Savitzky Golay filter using a $3^{\text {rd }}$ order polynomial for better visibility.

A transmitted beam spectrum for nitromethane acquired with 72000 counts and the scattered beam spectrum acquired with 1000 counts at $2 \theta=2.5^{\circ}$ are shown in Figure 6 a. and b, together with the data normalized with the transmitted beam at $2 \theta$ $=2.5^{\circ}$ and $2 \theta=6.5^{\circ}$ in c. and $\mathrm{d}$. The normalized spectra indicate that at photon energies above $\sim 100 \mathrm{keV}$ the count statistics are not sufficient to represent any meaningful data for the threat identification. Preliminary experiments performing EDXRD with common luggage objects e.g. liquids, laptops and books attenuating the beam indicate that only a limited number of photons with energies $<50 \mathrm{keV}$ reach the detector for certain luggage arrangements. For the EDXRD test data set we therefore only considered photons in the range of $50 \mathrm{keV}<\mathrm{E}>100 \mathrm{keV}$, corresponding to scattering vectors of 0.9 $-5.7 \AA^{-1}$
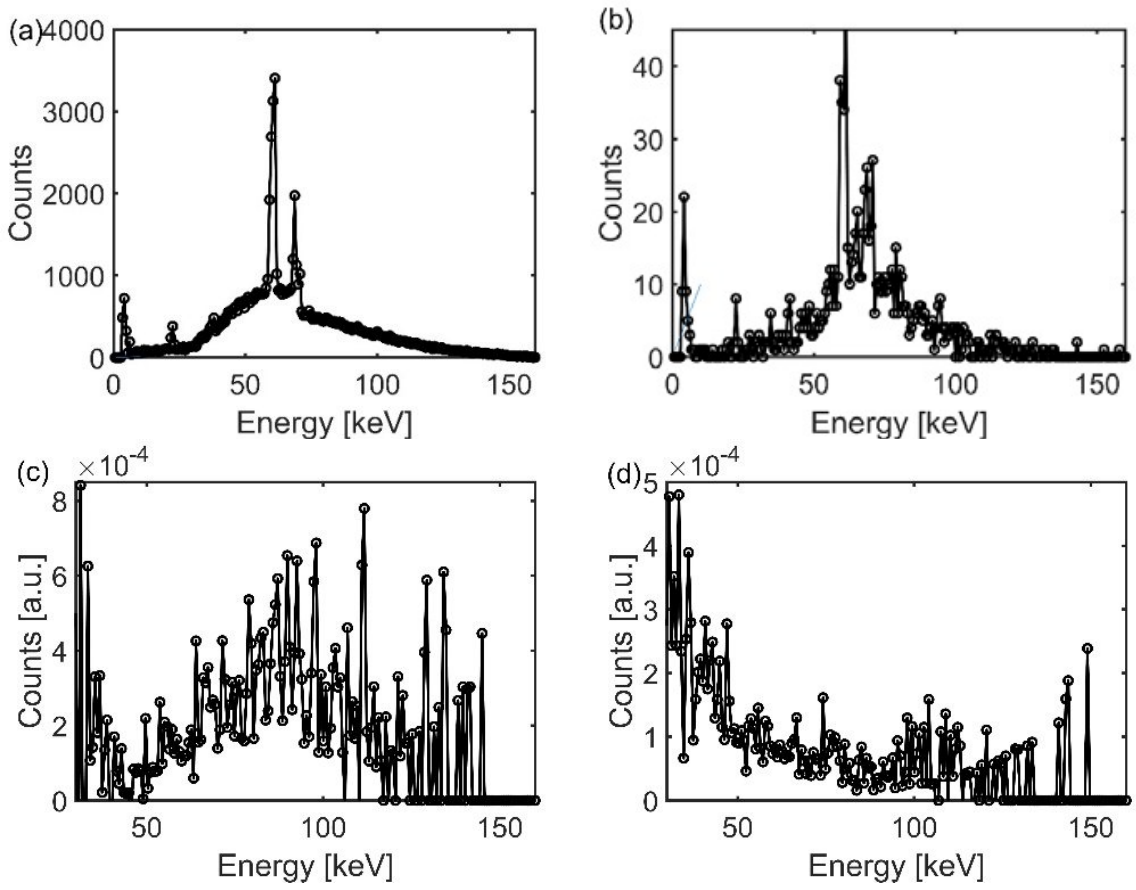

Figure 6. Illustration of a test data set of nitromethane with (a) the transmitted beam spectrum acquired with 72000 counts, (b) a scattered beam spectrum acquired with 1000 counts at $2 \theta=2.5^{\circ}$, in (c) and (d) the spectrum at $2 \theta=2.5^{\circ}$ and at $2 \theta=6.5^{\circ}$ respectively, normalized by the transmitted beam.

For extraction of the features from the diffraction patterns for the classification principle compound analysis [16] was performed, a commonly applied method for feature extraction from statistical poor EDXRD data [17]-[19]. For the initial testing of the threat detection the first 5 principle components were utilized. The classification was performed with a support vector machine [20] with a Gaussian radial basis function (RBF) using two hyper parameters: (1) the soft margin constraint 
parameter which allows to have some misclassified training examples and (2) the standard deviation to estimate the nonlinear space transformation. The model was calibrated by a series of repeated leave K-out experiments with $\mathrm{K}=10$. Utilizing the 10 repetitions of the 47 common liquids and the 11 liquid threats and randomly leaving out $\mathrm{K}=10$ enabled 57 training/test splits for a total of 570 test classifications. The results of the tests are shown in Figure 7.
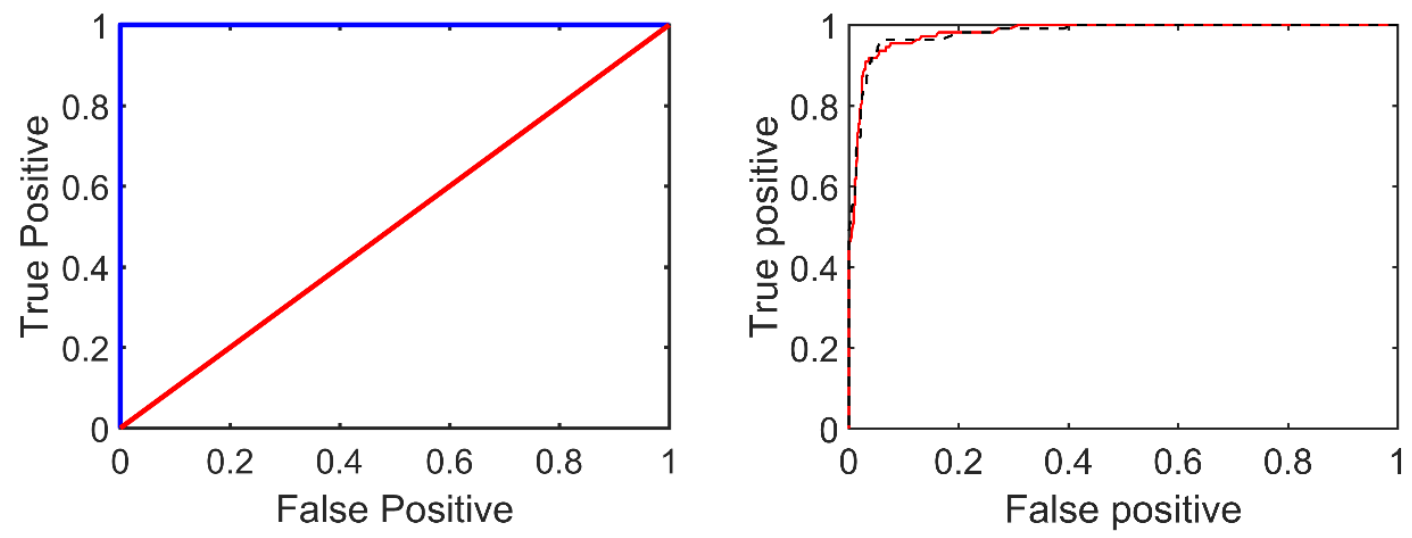

Figure 7. Illustration of classification performance limits showing perfect classification (-, blue) and random classification (-,red) (left ) . Performance of the classification for the test data set using SVM for unfiltered spectra (-,red) and Savitzky Golay filtered spectra (--,black) (right) the optimal performance of 95\% FP and 6\% TP is found at the point with least distance to the $(1,0)$ corner.

The classification was tested for unfiltered data and for data smoothed by applying a Savitzky-Golay filter using cubic function. The results indicate the feasibility of detection levels of $>95 \%$ true positive with $<6 \%$ false positive alarms. No significant effect of the filtering for the performance of the classification was observed and we conclude that filtering of the data can be omitted in the data processing for future experiments.

All EDXRD patterns collected in connection with the development of threat detection algorithms have been acquired from samples with a container diameters of $d=30-100 \mathrm{~mm}$ and the 11 threats were represented by liquid precursors which can be used for synthesis of explosives. However, the application for threat detection will also require the detection of explosives, among others also highly shock sensitive homemade and liquid explosives. Experimental samples in this range of common container sizes are not justifiable because of obvious safety concerns for the researcher and the research equipment. Drastic reductions of the sample volumes are therefore required, ideally a sample diameter far below the critical diameter of the substances. The critical diameter is the minimum diameter of a material required for a detonation, for the most prominent liquid explosives: methyl nitrate and nitroglycerine, the critical diameter is reported as $18 \mathrm{~mm}$ and $24 \mathrm{~mm}$ respectively [21].

The investigation of shock sensitive materials shall be performed in the smallest possible sample size. Therefore the feasibility of collecting diffraction signatures from material in thin walled glass capillary tubes (Mark tubes, Hilgenberg $\mathrm{GmbH}$, Malsfeld, Germany) was investigated. The tubes are commonly used in crystallography to probe powder samples.

For the tests glass capillaries with $d=2 \mathrm{~mm}$ were filled with sample volumes of $V=100 \mu \mathrm{l}$ and placed precisely in the center of rotation of the diffractometer with use of the motorized goniometerhead. The EDXRD patterns of nitromethane acquired in a capillary is shown together with the nitromethane reference sample in Figure 8. Both the scattering patterns from the bulk sample and the sample filled in the capillary show good agreement, although the acquisition time for collecting patterns with similar count statistics had to be increased substantial. However, the results clearly show the feasibility of investigating small amounts of substances which opens us the possibility to extend the threat detection algorithms to include liquid explosives. 


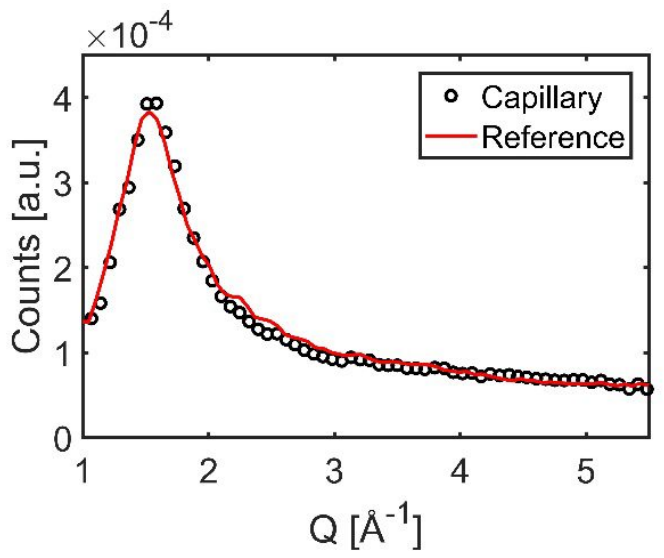

Figure 8. EDXRD pattern of nitromethane acquired in a $2 \mathrm{~mm}$ glass capillary (o, black) and reference (-, red) plotted as a function of $Q$.

\section{OUTLOOK}

The data set for testing the feasibility of classification of threats from materials commonly found in luggage will be extended by liquid explosives, homemade explosives (HME's) and solids, and different classifications algorithms will be investigated. The performance of the classification will be tested on different simulated luggage arrangements. In an iterative process, inspecting the performance of the classification, geometrical optimization will be performed to limit the number of detectors and determine the optimum required detector area at different angles.

\section{CONCLUSION}

A new non-commercial EDXRD was developed at the Department of Physics at the Technical University of Denmark. The validity of the acquired data and data processing routines has been confirmed by comparison with data acquired with superior count statistics from Synchrotron ADXRD. Accurate threat detection with $>95 \%$ true positive with $<6 \%$ false positive alarms can be obtained with limited statistics of 1000 counts per $2 \theta$ using principle component analysis and classification by a support vector machine.

\section{ACKNOWLEDGEMENTS}

The authors would like to thank Ove Rasmussen and Finn Saxild for the technical drawings and assistance by assembling the system. We acknowledge Anders Poulsen for assistance with the electric installations, Gerry Swisslow from Certified Scientific Software for integrations of new components in SPEC and Exruptive A/S and the Innovation Fund Demark for financial support.

\section{REFERENCES}

[1] Seidenstat, P., Splane, F. X., "Protecting airline passengers in the age of terrorism,” ABC-CLIO (2009)

[2] Eilbert, R. F., Krug, K. D., ”Aspects of image recognition in Vivid Technology's dual energy x-ray system for explosive detection," Proc. SPIE 1824, 129 - 143 (1992)

[3] O’Flynn, D., Reid, C. B., Christodoulou, C., Wilson, M. D., Veale, M. C., Seller, P., Hills, D., Desai, H., Wong, B., Speller, R., "Explosive detection using pixellated X-ray diffraction (PixD),” Jinst 8, P03007 (2013)

[4] Madden, R. R., Mahdavied, J., Smith, R. C., Sunramania, "An explosive detection system for airline security using coherent X-ray scattering technology,” Proc. SPIE, 7097, 707915-1 - 11 (2008)

[5] Harding, G., "X-ray diffraction imaging - A multi-generational perspective,” Appl. Radiat. Isotopes 67, 287 - 295 (2009) 
[6] Harding, G., Fleckenstein, H., Kosciesza, D., Olesinski,S., Strecker, H, Theedt, T., Zienert, G., "X-ray diffraction imaging with the Multiple Inverse Fan Beam topology: Principles, performance and potential for security screening." Appl. Radiat. Isotopes 70, 1228 - 1237 (2012)

[7] Skatter, S., Fritsch, S., Schlomka, J. B., ” Detecting Liquid Threats with X-ray Diffraction Imaging (XDi) using a Hybrid Approach to Navigate Trade-offs between Photon Count Statistics and Spatial Resolution,” Proc. SPIE 9847, 984704-1 - 14 (2016)

[8] Harding, G., Delfs, J., “Liquid identification with x-ray diffraction,” Proc. SPIE 6707, 67070T1-12 (2007)

[9] Harding, G., Fleckenstein, H., Olensinski, S., Zienert, G., "Liquid detection trail with x-ray diffraction,” Proc. SPIE 7806, 78060G-1-8 (2010)

[10] Luggar, R.D., Horroks, J. A., Speller, R. D., Lacey, R. J., “Low angle x-ray scatter for explosives detection: a geometry optimization,” Appl. Radiat. Isot. 48, 215 - 224 (1997)

[11] Crespy, C., Duvauchelle, P, Kaftandjan, V., Soulez, F., Ponard, P., "Energy dispersive x-ray diffraction to identify explosive substances: spectra analysis procedure optimization,” Nucl. Instr. Meth. Phys. Res. A 623, 1050 - 1060 (2010)

[12] Swisslow, G., “Certified Scientific Software,” <http://:www.certif.com> (5 July 2017)

[13] Brambilla, A., Ouvrier-Buffet, P., Gonon, G., Rinkel, J., Moulin, V., Boudou, C., and Verger, L., "Fast CdTe and CdZnTe Semiconductor Detector Arrays for Spectroscopic X-Ray Imaging," IEEE Trans. Nucl. Sci. 60(1), 408-415 (2013)

[14] Redus, H. R., Pantazis, J. A., Pantazis, T. J., Huber, A. C.; Cross, B. J., "Characterization of CdTe Detectors for Quantiative X-ray Spectroscopy”, IEEE Trans. Nucl. Sci. 56, 2524 - 2532 (2009)

[15] Savitzky, A., Golay, M. J. E., "Smoothing and Differentiation of Data by Simplified Least Squares Procedures," Analytical Chemistry 36, 1627-39 (1964)

[16] Jolliffe, I., "Principle Compound Analysis,” Springer Verlag, New York, (2002)

[17] Cook, E. J., Griffith, J. A., Koutalonis, M., Gent, C., Pani, S., Horroks, J. A., George, L., Hardwick, S., Speller, R., "Illicit drug detection using energy dispersive diffraction,” Proc. SPIE 7310, 73100I-1 - 10 (2009)

[18] Luggar, R. D., Farquharson, M. J., Horroks, J. A.m Lacey, R, J., "Multivariate analysis of statistically poor EDXRD spectra for the detection of concealed explosives,” X-ray Spectrom. 27, 87 - 94 (1998)

[19]Li, W., Zhong, Y., Qu, D., Sun, B., Li, M., Lui, J., ”Application of principle component analysis for identification of drugs packed in anthropomorphic phantom,” ARPN J. Eng. Appl. Sci. 7, 915 - 921 (2012)

[20] Cortes, C., Vapnik, V., "Support-vector networks," Machine Learning 20 (3), 273 - 297 (1995)

[21] Meyer, A. H. R., Köhler, J., “Explosives,” WILEY-VCH, Weinheim, (2007) 